Pacific Journal of Mathematic 


\title{
SOME EXPANSIONS INVOLVING BASIC HYPERGEOMETRIC FUNCTIONS OF TWO VARIABLES
}

\author{
V. K. JAIN
}

In this paper we obtain expansions of $q$-Appell type of functions of higher dimensions. These expansions are different in nature from the ones studies thus far. Transformations and reducibility of basic double hypergeometric functions are also discussed.

Burchnall and Chaundy [5, 6] has made a systematic study of the expansions of the Appell functions. Later on Jackson [8, 9] defined the $q$-analogue of Appell functions and made a parallel study by obtaining $q$-analogues of most of the results of Burchnall and Chaundy. Jackson [8; p. 78] had pointed out that it does not seem possible to obtain simple extensions of the expansions (46)-(51) of Burchnall and Chaundy [5]. In $\S 3$ of this paper we give $q$-analogues of five of the results, viz., (46)-(49) and (51) of Burchnall and Chaundy [5] cited above.

It may be remarked that Andrews [1] had proved that the $q$-analogue of Appell's function $F^{(1)}$ defined by Jackson [8] is infact reducible to the basic hypergeometric series ${ }_{3} \phi_{2}$. We show in $\S 4$ that some higher dimensional analogues of double hypergeometric functions could also be reduced to basic hypergeometric series and use the reduction formula for obtaining some interesting transformations for double hypergeometric functions. In this sequal we also derive $q$-analogues of some of the well known transformations of Appell functions and discuss their reducibility.

2. Definitions and notations. If we let

$$
\begin{aligned}
& {[a ; q]_{n}=(1-a)(1-a q) \cdots\left(1-a q^{n-1}\right),} \\
& {[a ; q]_{0}=1 \text { and }[a ; q]_{\infty}=\prod_{r=0}^{\infty}\left(1-a q^{r}\right),}
\end{aligned}
$$

then we may define the basic hypergeometric series as

$$
\begin{aligned}
{ }_{p+1} \phi_{p+r} & {\left[\begin{array}{l}
\left.a_{1}, a_{2}, \cdots, a_{p+1} ; q ; x\right] \\
b_{1}, b_{2}, \cdots, b_{p+r}
\end{array}\right] } \\
= & \sum_{n=0}^{\infty} \frac{\left[a_{1} ; q\right]_{n} \cdots\left[a_{p+1} ; q\right]_{n} x^{n}(-)^{n r} q^{r / 2(n-1)}}{[q ; q]_{n}\left[b_{1} ; q\right]_{n} \cdots\left[b_{p+r} ; q\right]_{n}}, \quad|q|<1,
\end{aligned}
$$

where the series ${ }_{p+1} \phi_{p+r}(x)$ converges for all positive integral values of $r$ and for all $x$, except when $r=0$, it converges only for $|x|<1$. 
Further to simplify writing we will abbreviate $[a ; q]_{n}$ by $[a]_{n}$ whenever there is no confusion regarding the base $q$.

Next, $q$-analogues of Appell functions $F^{(1)}, F^{(2)}, F^{(3)}$, and $F^{(4)}$ were defined by Jackson [8] as follows:

$$
\begin{aligned}
\phi^{(1)}\left[a: b, b^{\prime} ; c ; x, y ; q\right] & =\sum_{m=0}^{\infty} \sum_{n=0}^{\infty} \frac{[a]_{m+n}[b]_{m}\left[b^{\prime}\right]_{n} x^{m} y^{n}}{[q]_{m}[q]_{n}[c]_{m+n}}, \\
\phi^{(2)}\left[a: b, b^{\prime} ; c, c^{\prime} ; x, y ; q\right] & =\sum_{m=0}^{\infty} \sum_{n=0}^{\infty} \frac{[a]_{m+n}[b]_{m}\left[b^{\prime}\right]_{n} x^{m} y^{n}}{[q]_{m}[q]_{n}[c]_{m}\left[c^{\prime}\right]_{n}} \\
\phi^{(3)}\left[a, b ; a^{\prime}, b^{\prime} ; c ; x, y ; q\right] & =\sum_{m=0}^{\infty} \sum_{n=0}^{\infty} \frac{[a]_{m}[b]_{m}\left[a^{\prime}\right]_{n}\left[b^{\prime}\right]_{n} x^{m} y^{n}}{[q]_{m}[q]_{n}[c]_{m+n}} \\
\phi^{(4)}\left[a, b ; c, c^{\prime} ; x, y ; q\right] & =\sum_{m=0}^{\infty} \sum_{n=0}^{\infty} \frac{[a]_{m+n}[b]_{m+n} x^{m} y^{n}}{[q]_{m}[q]_{n}[c]_{m}\left[c^{\prime}\right]_{n}}
\end{aligned}
$$

Lastly, we define the generalized basic hypergeometric function iof two variables as:

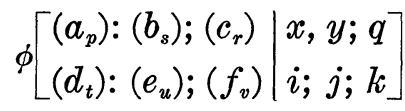

$$
\begin{aligned}
& =\sum_{m=0}^{\infty} \sum_{n=0}^{\infty} \frac{\left[\left(a_{p}\right)\right]_{m+n}\left[\left(b_{s}\right)\right]_{m}\left[\left(c_{r}\right)\right]_{n} x^{m} y^{n} q^{i m / 2(m-1)+j n / 2(n-1)+k m n}}{[q]_{m}[q]_{n}\left[\left(d_{t}\right)\right]_{m+n}\left[\left(e_{u}\right)\right]_{m}\left[\left(f_{v}\right)\right]_{n}} .
\end{aligned}
$$

3. In this section we give the $q$-analogues of the formulae of Burchnall and Chaundy (46, 47, 48, 49 and 51 of [5]):

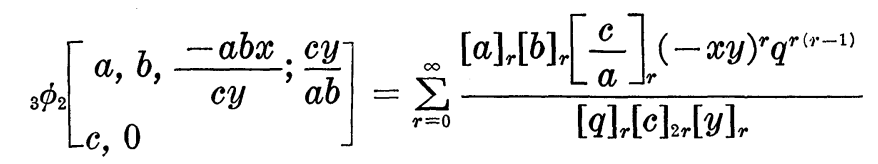

$$
\begin{aligned}
& \times \phi\left[\begin{array}{c|c}
a q^{r}: b q^{r} ; b q^{r} & x q^{r}, \frac{c y}{a b} ; q \\
c q^{2 r}: y q^{r} ;- & 1 ;-;-
\end{array}\right] \\
& \phi\left[\begin{array}{l|l}
a: b ; b & \frac{c x}{a b}, \frac{c y}{a b} ; q \\
c:-x ;- & 1 ;-;-
\end{array}\right]=\sum_{r=0}^{\infty} \frac{[a]_{r}[b]_{r}\left[\frac{c}{a}\right]_{r}(c x y)^{r} q^{r / 2(r-1)}}{[q]_{r}[c]_{2 r}[-x]_{r}(a b)^{r}} \\
& \times_{3} \phi_{2}\left[\begin{array}{l}
a q^{r}, b q^{r}, \frac{-x}{y} q^{r} ; \frac{c y}{a b} \\
c q^{2 r},-x q^{r}
\end{array}\right], \\
& { }_{3} \phi_{2}\left[\begin{array}{l}
\left.a, b, \frac{-x}{y} ; y\right] \\
c, 0
\end{array}\right]=\sum_{r=0}^{\infty} \frac{[a]_{r}[b]_{r}\left[\frac{a b}{c}\right]_{r}(c x y)^{r} q^{r / 2(5 r-3)}}{[q]_{r}[c]_{2 r}[y]_{r}}
\end{aligned}
$$




$$
\begin{aligned}
& \times \phi\left[\begin{array}{l|c}
-: a q^{r}, b q^{r} ; a q^{r}, b q^{r} & x q^{2 r}, y q^{r} ; q \\
c q^{2 r}: y q^{r} ;- & 1 ;-; 1
\end{array}\right], \\
& \phi\left[\begin{array}{l|l}
-: a, b ; a, b & x, y ; q \\
c: y ;- & 1 ;-; 1
\end{array}\right]=\sum_{r=0}^{\infty} \frac{\left[a \left[_{r}[b]_{r}\left[\frac{c}{a b}\right]_{r}(a b x y)^{r} q^{r / 2(5 r-3)}\right.\right.}{[q]_{r}[c]_{2 r}[y]_{r}} \\
& \times{ }_{3} \phi_{2}\left[\begin{array}{l}
a q^{r}, b q^{r}, \frac{-x q^{r}}{y} ; y q^{r} \\
c q^{2 r}, 0
\end{array}\right]
\end{aligned}
$$

and

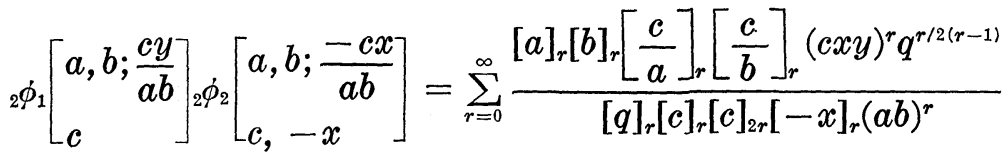

$$
\begin{aligned}
& \times{ }_{3} \phi_{2}\left[\begin{array}{l}
a q^{r}, b q^{r}, \frac{-x}{y} q^{r} ; \frac{c y}{a b} \\
c q^{2 r},-x q^{r}
\end{array}\right] .
\end{aligned}
$$

Proof of (3.1). In view of the $q$-analogue of Gauss' summation theorem $[12 ; 3.3 .2 .5]$ we have

$$
\frac{\left[\frac{c}{b}\right]_{n}\left[\frac{c}{b}\right]_{m} q^{m n}}{\left[\frac{c}{b}\right]_{m+n}}={ }_{2} \phi_{1}\left[\begin{array}{l}
q^{-m}, q^{-n} ; \frac{b q}{c} \\
\frac{b}{c} q^{1-m-n}
\end{array}\right],
$$

multiplying both sides of (3.6) by

$$
\frac{[a]_{m}[b]_{m}\left[\frac{c}{a}\right]_{n}\left[\frac{c}{b}\right]_{m+n} x^{m} y^{n} q^{m / 2(m-1)}}{[q]_{m}[q]_{n}[c]_{m+n}[y]_{m}\left[\frac{c}{b}\right]_{m}}
$$

and summing with respect to $m$ and $n$ from 0 to $\infty$, we get:

$$
\begin{aligned}
\sum_{m=0}^{\infty} \frac{[a]_{m}[b]_{m} x^{m} q^{m / 2(m-1)}}{[q]_{m}[c]_{m}[y]_{m}}{ }_{2} \phi_{1}\left[\frac{c}{a}, \frac{c}{b} ; y q^{m}\right] \\
=\sum_{r=0}^{\infty} \frac{[a]_{r}[b]_{r}\left[\frac{c}{a}\right]_{r}(-x y)^{r} q^{r(r-1)}}{[q]_{r}[c]_{2 r}[y]_{r}} \\
\quad \times \sum_{m=0}^{\infty} \frac{\left[a q^{r}\right]_{m}\left[b q^{r}\right]_{m} x^{m} q^{m / 2(m+2 r-1)}}{[q]_{m}\left[c q^{2 r}\right]_{m}\left[y q^{r}\right]_{m}}{ }_{2} \phi_{1}\left[\frac{c}{a} q^{r}, \frac{c}{b} q^{r+m} ; y\right] .
\end{aligned}
$$

Using the transformation [11]: 


$$
{ }_{2} \phi_{1}\left[\begin{array}{l}
a, b ; x \\
e
\end{array}\right]=\frac{\left[\frac{a b x}{e}\right]_{\infty}}{[x]_{\infty}} \phi_{1}\left[\frac{e}{a}, \frac{e}{b} ; \frac{a b x}{e}\right],
$$

to transform the inner ${ }_{2} \phi_{1}$ on both sides of (3.7) and simplifying, we get (3.1).

Proof of (3.2). The $q$-analogue of Vandermonde's theorem [12; 3.3.2.7] may be rewritten as:

$$
\frac{\left[\frac{c}{b}\right]_{m+n}}{\left[\frac{c}{b}\right]_{m}\left[\frac{c}{b}\right]_{n}}=q_{2{ }_{2} \phi_{1}}\left[\frac{q^{-m}}{b}, q^{-n} ; q\right] .
$$

On multiplying both sides of (3.9) by

$$
\frac{[a]_{m}[b]_{m}\left[\frac{c}{a}\right]_{n}\left[\frac{c}{b}\right]_{n}(c x)^{m} y^{n} q^{m / 2(m-1)}}{[q]_{m}[q]_{n}[c]_{m+n}[-x]_{m}(a b)^{m}}
$$

and summing with respect to $m$ and $n$ from 0 to $\infty$, we have:

$$
\begin{aligned}
\sum_{m=0}^{\infty} \frac{[a]_{m}[b]_{m}(c x)^{m} q^{m / 2(m-1)}}{[q]_{m}[c]_{m}[-x]_{m}(a b)^{m}}{ }_{2} \phi_{1}\left[\frac{c}{a}, \frac{c}{b} q^{m} ; y\right] \\
=\sum_{r=0}^{\infty} \frac{[a]_{r}[b]_{r}\left[\frac{c}{a}\right]_{r}(c x y)^{r} q^{r / 2(r-1)}}{\left[q_{r}\right][c]_{2 r}[-x]_{r}(a b)^{r}} \sum_{m=0}^{\infty} \frac{\left[a q^{r}\right]_{m}\left[b q^{r}\right]_{m}(c x)^{m} q^{m / 2(m-1+2 r)}}{[q]_{m}\left[c q^{2 r}\right]_{m}\left[-x q^{r}\right]_{m}(a b)^{m}} \\
\quad \times{ }_{2} \phi_{1}\left[\frac{c}{a} q^{r} \frac{c}{b} q^{r} ; y q^{m}\right], \\
c q^{m+2 r}
\end{aligned}
$$

transforming the two ${ }_{2} \phi_{1}$ in (3.10), using (3.8), we get:

$$
\begin{aligned}
& \phi\left[\begin{array}{l|l}
a: b ; b & \frac{c x}{a b}, \frac{c y}{a b} ; q \\
c:-x ;- & 1 ;-;-
\end{array}\right]=\sum_{r=0}^{\infty} \frac{[a]_{r}[b]_{r}\left[\frac{c}{a}\right]_{r}(c x y)^{r} q^{r / 2(r-1)}}{[q]_{r}[c]_{2 r}[-x]_{r}(a b)^{r}} \\
& \times \sum_{m=0}^{\infty} \sum_{n=0}^{\infty} \frac{\left[a q^{r}\right]_{m+n}\left[b q^{r}\right]_{m+n}[y]_{m}(c x)^{m}(c y)^{n} q^{m / 2(m+2 r-1)}}{[q]_{m}[q]_{n}\left[c q^{2 r}\right]_{m+n}\left[-x q^{r}\right]_{m}(a b)^{m+n}} \\
& =\sum_{r=0}^{\infty} \frac{[a]_{r}[b]_{r}\left[\frac{c}{a}\right]_{r}(c x y)^{r} q^{r / 2(r-1)}}{[q]_{r}[c]_{2 r}[-x]_{r}(a b)^{r}} \sum_{n=0}^{\infty} \frac{\left[a q^{r}\right]_{n}\left[b q^{r}\right]_{n}(c y)^{n}}{[q]_{n}\left[c q^{2 r}\right]_{n}(a b)^{n}} \\
& \times{ }_{2} \phi_{1}\left[\begin{array}{l}
q^{-n}, y ; \frac{-x}{y} q^{r+n} \\
-x q^{r}
\end{array}\right] \text {. }
\end{aligned}
$$


(3.11) gives (3.2), on summing the inner most series by the $q$-analogue of Gauss' theorem.

Proof of (3.3). On the other hand if we start with the $q$-analogue of the Saalschütz summation theorem [12; 3.3.2.2]

$$
\frac{[b]_{m+n}\left[\frac{c}{a}\right]_{m}\left[\frac{c}{a}\right]_{n}}{[b]_{m}[b]_{n}\left[\frac{c}{a}\right]_{m+n}}={ }_{3} \phi_{2}\left[\begin{array}{l}
q^{-m}, q^{-n}, \frac{a b}{c} ; q \\
b, \frac{a}{c} q^{1-m-n}
\end{array}\right]
$$

and proceed as in the proof of (3.1), we obtain:

$$
\begin{aligned}
\sum_{m=0}^{\infty} \frac{[a]_{m}[b]_{m} x^{m} q^{m / 2(m-1)}}{[q]_{m}[c]_{m}[b y]_{m}}{ }_{2} \phi_{2}\left[\begin{array}{l}
b q^{m}, \frac{c}{a} ; a y q^{m} \\
c q^{m}, b y q^{m}
\end{array}\right] & \sum_{m=0}^{\infty} \frac{\left[a q^{r}\right]_{m}\left[b q^{r}\right]_{m} x^{m} q^{m / 2(m+4 r-1)}}{[q]_{m}\left[c q^{2 r}\right]_{m}\left[b y q^{2 r}\right]_{m}} \\
= & \sum_{r=0}^{\infty} \frac{[a]_{r}[b]_{r}\left[\frac{a b}{c}\right]_{r}(c x y)^{r} q^{r / 2(5 r-3)}}{[q]_{r}[c]_{2 r}[b y]_{2 r}} \\
& \times{ }_{2} \phi_{2}\left[\begin{array}{l}
b q^{r}, \frac{c}{a} q^{m+r} ; a y q^{2 r+m} \\
c q^{m+2 r}, b y q^{m+2 r}
\end{array}\right] .
\end{aligned}
$$

Transforming the inner ${ }_{2} \phi_{2}$ on both sides by the following formula of Jackson [7]:

$$
{ }_{2} \phi_{2}\left[\begin{array}{l}
a, b ; \frac{c z}{b} \\
c, a z
\end{array}\right]=\frac{[z]_{\infty}}{[a z]_{\infty}}{ }_{2} \phi_{1}\left[\begin{array}{l}
a, \frac{c}{b} ; z \\
c
\end{array}\right]
$$

we get (3.3) on some reduction.

Proof of (3.4). To accomplishes (3.4) we need only to start with

$$
\frac{\left[\frac{c}{b}\right]_{m+n}[a]_{m}[a]_{n}}{\left[\frac{c}{b}\right]_{m}\left[\frac{c}{b}\right]_{n}[a]_{m+n}}={ }_{3} \phi_{2}\left[\begin{array}{l}
q^{-m}, q^{-n}, \frac{c}{a b} ; q \\
\frac{c}{b}, \frac{1}{a} q^{1-m-n}
\end{array}\right],
$$

instead of (3.12) and proceed along the lines of proof of (3.3).

Proof of (3.5). Lastly, starting with (3.9) with $b=1$, multiplying both sides by $[a]_{m}[b]_{m}[c / a]_{n}[c / b]_{n}(c x)^{m} y^{n} q^{m / 2(m-1)} /[q]_{m}[q]_{n}[c]_{m+n}[-x]_{m}(a b)^{m}$ and summing with respect to $m$ and $n$ from 0 to $\infty$, we get

$$
{ }_{2} \phi_{1}\left[\frac{c}{a}, \frac{c}{b} ; y\right]{ }_{2} \phi_{2}\left[\begin{array}{l}
a, b ; \frac{-c x}{a b} \\
c,-x
\end{array}\right]=\sum_{r=0}^{\infty} \frac{[a]_{r}[b]_{r}\left[\frac{c}{a}\right]_{r}\left[\frac{c}{b}\right]_{r}(c x y)^{r} q^{r / 2(r-1)}}{[q]_{r}[c]_{r}[c]_{2 r}[-x]_{r}(a b)^{r}}
$$




$$
\times \sum_{m=0}^{\infty} \frac{\left[a q^{r}\right]_{m}\left[b q^{r}\right]_{m}(c x)^{m} q^{m / 2(m+2 r-1)}}{[q]_{m}\left[c q^{2 r}\right]_{m}\left[-x q^{r}\right]_{m}(a b)^{m}}{ }_{2} \phi_{1}\left[\begin{array}{c}
\frac{c}{a} q^{r}, \frac{c}{b} q^{r} ; y q^{m} \\
c q^{2 r+m}
\end{array}\right] .
$$

Transforming the ${ }_{2} \dot{\phi}_{1}$ by (3.8), we get:

$$
\begin{aligned}
{ }_{2} \phi_{1}\left[\begin{array}{c}
a, b \\
c
\end{array} \frac{c y}{a b}\right]_{2} \phi_{2}\left[\begin{array}{l}
\left.a, b ; \frac{-c x}{a b}\right] \\
c,-x
\end{array}\right]=\sum_{r=0}^{\infty} \frac{[a]_{r}[b]_{r}\left[\frac{c}{a}\right]_{r}\left[\frac{c}{b}\right]_{r}(c x y)^{r} q^{r / 2(r-1)}}{[q]_{r}[c]_{r}[c]_{2 r}[-x]_{r}(a b)^{r}} \\
\quad \times \sum_{m=0}^{\infty} \frac{\left[a q^{r}\right]_{m}\left[b q^{r}\right]_{m}[y]_{m}(c x)^{m} q^{m / 2(m+2 r-1)}}{[q]_{m}\left[c q^{2 r}\right]_{m}\left[-x q^{r}\right]_{m}(a b)^{m}}\left[a q^{r+m}, b q^{r+m} ; \frac{c y}{a b}\right] \\
c q^{m+2 r} \\
=\sum_{r=0}^{\infty} \frac{[a]_{r}[b]_{r}\left[\frac{c}{a}\right]_{r}\left[\frac{c}{b}\right]_{r}(c x y)^{r} q^{r / 2(r-1)}}{[q]_{r}[c]_{r}[c]_{2 r}[-x]_{r}(a b)^{r}} \sum_{n=0}^{\infty} \frac{\left[a q^{r}\right]_{n}\left[b q^{r}\right]_{n}(c y)^{n}}{[q]_{n}\left[c q^{2 r}\right]_{n}(a b)^{n}} \\
\quad \times{ }_{2} \phi_{1}\left[q^{-n}, y ; \frac{-x}{y} q^{r+n}\right] . \\
-x q^{r}
\end{aligned}
$$

(3.15) gives (3.5), on summing the inner most series by the $q$-analogue of Gauss' theorem.

4. In this section we would prove $q$-analogues of the transformations between the various Appell functions. In fact we begin by proving:

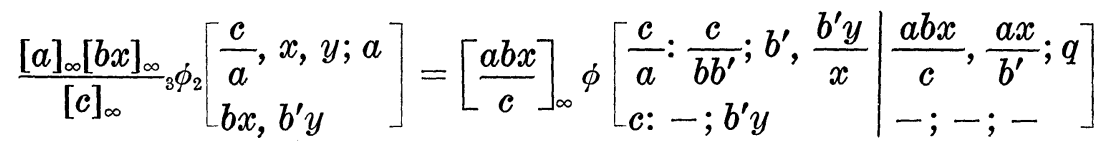

$$
\begin{aligned}
& =[x]_{\infty} \phi\left[\begin{array}{l|l}
-: a, b ; \frac{c}{a}, b^{\prime} & x,-a y ; q \\
c:-; b^{\prime} y & -; 1 ; 1
\end{array}\right] .
\end{aligned}
$$

Proof of (4.1). If we denote the right hand side of (4.1) by $S$, then

$$
S=\left[\frac{a b x}{c}\right]_{\infty} \sum_{n=0}^{\infty} \frac{\left[\frac{c}{a}\right]_{n}\left[b^{\prime}\right]_{n}\left[\frac{b^{\prime} y}{x}\right]_{n}(a x)^{n}}{[q]_{n}\left[b^{\prime} y\right]_{n}[c]_{n}\left(b^{\prime}\right)^{n}}{ }_{2} \phi_{1}\left[\frac{c}{a} q^{n}, \frac{c}{b b^{\prime}} ; \frac{a b x}{c}\right]
$$

Transforming the inner ${ }_{2} \phi_{1}$ by (3.13) and rearranging the series, we get 


$$
S=\left[\frac{a x}{b^{\prime}}\right]_{\infty} \sum_{m=0}^{\infty} \frac{[a]_{m}\left[\frac{c}{b b^{\prime}}\right]_{m}(-b x)^{m} q^{m / 2(m-1)}}{[q]_{m}[c]_{m}\left[\frac{a x}{b^{\prime}}\right]_{m}} \phi_{2}\left[\frac{c}{a}, \frac{b^{\prime} y}{x}, b^{\prime} ; \frac{a x}{b^{\prime}} q^{m}\right]
$$

Now transforming the inner ${ }_{3} \phi_{2}$ using:

$$
{ }_{3} \phi_{2}\left[\begin{array}{l}
a, b, c ; \frac{e g}{a b c} \\
e, g
\end{array}\right]=\Pi\left[\begin{array}{l}
\frac{g}{c}, \frac{e g}{a b} \\
g, \frac{e g}{a b c}
\end{array}\right]{ }_{3} \dot{\phi}_{2}\left[\begin{array}{l}
\frac{e}{a}, \frac{e}{b}, c ; \frac{g}{c} \\
e, \frac{e g}{a b}
\end{array}\right],
$$

(which is obtained from [11; (8.3)] by taking the limit as $N \rightarrow \infty$ ) we get,

$$
\begin{aligned}
S & =\frac{[y]_{\infty}[a x]_{\infty}}{\left[b^{\prime} y\right]_{\infty}} \sum_{m=0}^{\infty} \frac{[a]_{m}\left[\frac{c}{b b^{\prime}}\right]_{m}(-b x)^{m} q^{m / 2(m-1)}}{[q]_{m}[c]_{m}[a x]_{m}}{ }_{3} \phi_{2}\left[\begin{array}{l}
\left.a q^{m}, \frac{c x}{b^{\prime} y} q^{m}, b^{\prime} ; y\right] \\
c q^{m}, a x q^{m}
\end{array}\right] \\
& =\frac{[y]_{\infty}[a x]_{\infty}}{\left[b^{\prime} y\right]_{\infty}} \sum_{m=0}^{\infty} \frac{[a]_{m}\left[\frac{c}{b b^{\prime}}\right]_{m}(-b x)^{m} q^{m / 2(m-1)}}{[q]_{m}[c]_{m}[a x]_{m}}{ }_{3} \phi_{2}\left[\begin{array}{l}
q^{-m}, \frac{b^{\prime} y}{c x} q^{1-m}, b^{\prime} ; q \\
\frac{b b^{\prime}}{c} q^{1-m}, 0
\end{array}\right] .
\end{aligned}
$$

Next, transforming the inner ${ }_{3} \phi_{2}$ by $[14]$

$$
{ }_{2} \dot{\phi}_{1}\left[\begin{array}{l}
a, b ; \frac{e c}{a b} \\
e
\end{array}\right]=\Pi\left[\begin{array}{l}
\frac{e}{a}, \frac{e}{b} ; \\
e, \frac{e}{a b}
\end{array}\right]{ }_{3} \phi_{2}\left[\begin{array}{l}
a, b, c ; q \\
\frac{a b q}{e}, 0
\end{array}\right],
$$

(provided either $a, b$ or $c$ is of the form $q^{-N}, N$ a nonnegative inner, if only $c=q^{-N}$ then $|e c / a b|<1$ ) we have

$$
\begin{aligned}
S & =\frac{[y]_{\infty}[a x]_{\infty}}{\left[b^{\prime} y\right]_{\infty}} \sum_{m=0}^{\infty} \frac{[a]_{m}\left[\frac{c}{b}\right]_{m}(-b x)^{m} q^{m / 2(m-1)}}{[q]_{m}[c]_{m}[a x]_{m}}{ }_{2} \phi_{1}\left[\frac{c}{b} q^{-m}, b^{\prime} ; \frac{y q}{b x}\right] \\
& =\frac{[y]_{\infty}[a x]_{\infty}}{\left[b^{\prime} y\right]_{\infty}} \sum_{n=0}^{\infty} \frac{[a]_{n}\left[b^{\prime}\right]_{n} y^{n}}{[q]_{n}[c]_{n}[a x]_{n}}{ }_{2} \phi_{2}\left[\begin{array}{l}
a q^{n}, \frac{c}{b} q^{n} ; b x \\
c q^{n}, a x q^{n}
\end{array}\right],
\end{aligned}
$$

once again, transforming ${ }_{2} \phi_{2}$ by (3.13), we get

$$
S=\frac{[x]_{\infty}[y]_{\infty}}{\left[b^{\prime} y\right]_{\infty}} \sum_{m=0}^{\infty} \sum_{n=0}^{\infty} \frac{[a]_{m+n}[b]_{m}\left[b^{\prime}\right]_{n} x^{m} y^{n}}{[q]_{m}[q]_{n}[c]_{m+n}}
$$

Lastly, an appeal to 


$$
\phi^{(1)}\left[a: b, b^{\prime} ; c ; x, y ; q\right]=\Pi\left[\begin{array}{l}
a, b x, b^{\prime} y \\
c, x, y
\end{array}\right]_{3} \phi_{2}\left[\begin{array}{l}
\frac{c}{a}, x, y ; a \\
b x, b^{\prime} y
\end{array}\right],
$$

due to Andrews [1], yields (4.1).

One the other hand in order to prove (4.2), we need only rewrite (4.5) in the form

$$
S=\frac{[y]_{\infty}[x]_{\infty}}{\left[b^{\prime} y\right]_{\infty}} \sum_{m=0}^{\infty} \frac{[a]_{m}[b]_{m} x^{m}}{[q]_{m}[c]_{m}}{ }_{2} \phi_{1}\left[\begin{array}{c}
a q^{m}, b^{\prime} ; y \\
c q^{m}
\end{array}\right]
$$

and transform the inner ${ }_{2} \dot{\phi}_{1}$ by (3.13).

It may be observed that the ${ }_{3} \phi_{2}$ in (4.1) remains unchanged if we interchange the roles of $x$ and $y$ and that of $b$ and $b^{\prime}$. This yields us the transformation:

$$
\begin{aligned}
& {\left[\frac{a b x}{c}\right]_{\infty}\left[b^{\prime} y\right]_{\infty} \phi\left[\begin{array}{l|l}
\frac{c}{a}: \frac{c}{b b^{\prime}} ; b^{\prime}, \frac{b^{\prime} y}{x} & \frac{a b x}{c}, \frac{a x}{b^{\prime}} ; q \\
c:-; b^{\prime} y & -;-;-
\end{array}\right]} \\
& =\left[\frac{a b^{\prime} y}{c}\right]_{\infty}[b x]_{\infty} \phi\left[\begin{array}{l}
\frac{c}{a}: \frac{c}{b b^{\prime}} ; b, \frac{b x}{y} \mid \frac{a b^{\prime} y}{c}, \frac{a y}{b} ; q \\
c:-; b x
\end{array}\right]
\end{aligned}
$$

which is a $q$-analogue of a known transformation between two $F^{(1)}$ 's [3; $\S 9.4$ (4) and (5)] to which it reduces if we replace $a, b, b^{\prime}, c$ by $q^{a}, q^{b}, q^{b^{\prime}}, q^{c}$ and let $q \rightarrow 1$. Similarly we could have obtained from (4.2) the transformation

$$
\begin{aligned}
& \frac{\left[b^{\prime} y\right]_{\infty}}{[y]_{\infty}} \phi\left[\begin{array}{l|l}
-: a, b ; \frac{c}{a}, b^{\prime} & x,-a y ; q \\
c:-; b^{\prime} y & -; 1 ; 1
\end{array}\right] \\
& \quad=\frac{[b x]_{\infty}}{[x]_{\infty}} \phi\left[\begin{array}{l}
-: a, b^{\prime} ; \frac{c}{a}, b \\
c:-; b x
\end{array} \mid \begin{array}{l}
y,-a x ; q \\
-; 1 ; 1
\end{array}\right],
\end{aligned}
$$

which is the $q$-analogue of yet another known transformation between two $F^{(3)}$ 's [3] to which it reduces if we replace $a, b, b^{\prime}, c$ by $q^{a}, q^{b}, q^{b^{\prime}}, q^{c}$ and let $q \rightarrow 1$.

Next, we would prove:

$$
\begin{aligned}
& \frac{[a y]_{\infty}}{[y]_{\infty}} \phi\left[\begin{array}{l|l}
a: b ; \frac{c^{\prime}}{b^{\prime}} & x,-b^{\prime} y ; q \\
a y: c ; c^{\prime} & -; 1 ;-
\end{array}\right]=\phi^{(2)}\left[a: b, b^{\prime} ; c, c^{\prime} ; x, y ; q\right] \\
& =\frac{[a x]_{\infty}}{[x]_{\infty}} \phi\left[\begin{array}{l|l}
a: b^{\prime} ; \frac{c}{b} & y,-b x ; q \\
a x: c^{\prime} ; c & -; 1 ;-
\end{array}\right],
\end{aligned}
$$




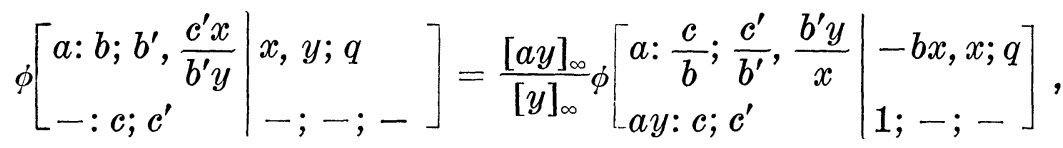

$$
\begin{aligned}
& \phi\left(\begin{array}{l|l}
a: b,-b ; c,-c & \frac{x q}{y},-y ; q \\
-: b^{2}, a x, \frac{q}{y} ; c^{2}, a y & 1 ;-;-1
\end{array}\right) \\
& =\Pi\left[\begin{array}{l}
x, y \\
a x, a y
\end{array}\right] \phi\left[\begin{array}{l}
a, a q:-; \frac{q}{x}, \frac{q^{2}}{x} \\
x^{2}, \frac{x^{2} y^{2}}{q^{3}} ; q^{2} \\
-; b^{2} q ; c^{2} q
\end{array}\right]
\end{aligned}
$$

(provided $a$ is of the form $q^{-N}$ )

and

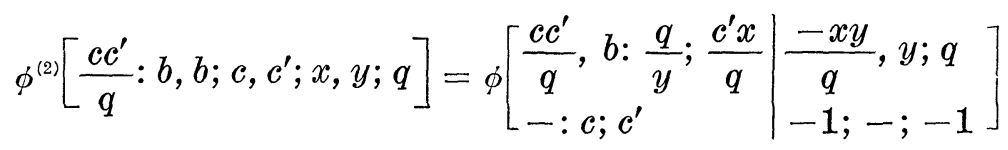

(provided $c c^{\prime} / q$ is of the form $q^{-N}$ ).

Proof of (4.7-4.8). Rewriting the left hand side in the form

$$
\frac{[a y]_{\infty}}{[y]_{\infty}} \sum_{m=0}^{\infty} \frac{[a]_{m}[b]_{m} x^{m}}{[q]_{m}[c]_{m}[a y]_{m}{ }^{2}} \phi_{2}\left[\begin{array}{l}
a q^{m}, \frac{c^{\prime}}{b^{\prime}} ; b^{\prime} y \\
c^{\prime}, a y q^{m}
\end{array}\right]
$$

and transforming ${ }_{2} \phi_{2}$ by (3.13) we get the right hand side of (4.7), for proving (4.8) rearranging the series on the right hand side of (4.7) and transforming the ${ }_{2} \phi_{1}$ by (3.13). This reduces to a known transformation between $F^{(2)}$ 's $[3 ; \S(9.4)]$. It may be pointed out that (4.7) is a known result due to Upadhyay [13].

Proof of (4.9). Rewrite the left hand side of (4.9) as:

$$
S=\sum_{n=0}^{\infty} \frac{[a]_{n}\left[b^{\prime}\right]_{n}\left[\frac{c^{\prime} x}{b^{\prime} y}\right]_{n} y^{n}}{[q]_{n}\left[c^{\prime}\right]_{n}}{ }_{2} \phi_{1}\left[\begin{array}{c}
a q^{n}, b ; x \\
c
\end{array}\right],
$$

transforming the ${ }_{2} \dot{\phi}_{1}$ by (3.13) and rearranging the series, we get

$$
S=\frac{[a x]_{\infty}}{[x]_{\infty}} \sum_{m=0}^{\infty} \frac{[a]_{m}\left[\frac{c}{b}\right]_{m}(-b x)^{m} q^{m / 2(m-1)}}{[q]_{m}[c]_{m}[a x]_{m}}{ }_{3} \phi_{2}\left[\begin{array}{l}
\left.b^{\prime}, \frac{c^{\prime} x}{b^{\prime} y}, a q^{m} ; y\right] \\
c^{\prime}, a x q^{m}
\end{array}\right] .
$$

Transforming the ${ }_{3} \dot{\phi}_{2}$ by (4.2) and simplifying, we get (4.9). This result is a $q$-analogue of a known transformation between two $F^{(2)}$ 's [3]. 
Proof of (4.10). If we denote the left hand side of (4.10) by $S$, then

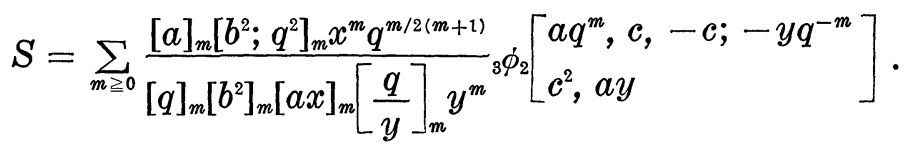

Transforming the ${ }_{3} \phi_{2}$ by the formula [10; equation 3.1]:

$$
{ }_{3} \phi_{2}\left[\begin{array}{l}
a, b,-b ;-z \\
b^{2}, a z
\end{array}\right]=\frac{[z]_{\infty}}{[a z]_{\infty}}{ }_{2} \phi_{1}\left[\begin{array}{l}
a, a q ; q^{2} ; z^{2} \\
b^{2} q
\end{array}\right]
$$

and rearranging the series, we get

$$
S=\frac{[y]_{\infty}}{[a y]_{\infty}} \sum_{n \geq 0} \frac{[a]_{2 n} y^{2 n}}{\left[q^{2} ; q^{2}\right]_{n}\left[c^{2} q ; q^{2}\right]_{n}{ }^{3}} \phi_{2}\left[\begin{array}{l}
a q^{2 n}, b,-b ;-x q^{-2 n} \\
b^{2}, a x
\end{array}\right] .
$$

Once again, transforming the ${ }_{3} \phi_{2}$ by (4.12) and simplifying, (4.13) yields (4.10), which is the $q$-analogue of a terminating version of [4; (4.7)].

Proof of (4.11). In view of the $q$-analogue of Gauss' summation theorem, we have

$$
\frac{[b]_{m}[b]_{n}}{[b]_{m+n}}=q_{2}^{-m n} \phi_{1}\left(\begin{array}{l}
q^{-m}, q^{-n} ; \frac{q}{b} \\
\frac{1}{b} q^{1-m-n}
\end{array}\right) .
$$

Multiplying both sides by $\left[c c^{\prime} / q\right]_{m+n}[b]_{m+{ }_{n}} x^{m} y^{n} /[q]_{m}[q]_{n}[c]_{m}\left[c^{\prime}\right]_{n}$ (where $c c^{\prime}\left(q=q^{-N}\right)$ and summing with respect to $m$ and $n$, we get

$$
\begin{aligned}
& \phi^{(2)}\left[\frac{c c^{\prime}}{q}: b, b ; c, c^{\prime} ; x, y ; q\right] \\
& =\sum_{m \geq 0} \sum_{n \geqq 0} \sum_{p \geq 0} \frac{\left[\frac{c c^{\prime}}{q}\right]_{m+n+2 p}[b]_{m+n+p} x^{m+p} y^{n+p}(-)^{p} q^{-p / 2(p+1+2 n+2 m)}}{[q]_{m}[q]_{n}[q]_{p}[c]_{m+p}\left[c^{\prime}\right]_{n+p} q^{m n}} \\
& =\sum_{m \geqq 0} \sum_{n \geqq 0} \sum_{p \geq 0} \frac{\left[\frac{c c^{\prime}}{q}\right]_{m+n+p}[b]_{m+n+p} x^{m+p} y^{n+p}\left(-c^{\prime}\right)^{p} q^{p^{2 / 2-3 p / 2}}}{[q]_{m}[q]_{n}[q]_{p}[c]_{m}\left[c^{\prime}\right]_{n+p} q^{m+m p}} \sum_{s=0}^{p} \frac{\left[q^{-p}\right]_{s}\left[\frac{1}{c^{\prime}} q^{1-n-p}\right]_{s} q^{s}}{[q]_{s}\left[c q^{m}\right]_{s}} \\
& =\sum_{m \geq 0} \sum_{n \geq 0} \frac{\left[\frac{c c^{\prime}}{q}\right]_{m+n}[b]_{m+n} x^{m} y^{n}}{[q]_{m}[q]_{n}[c]_{m}\left[c^{\prime}\right]_{n} q^{m n}{ }_{1}} \phi_{0}\left[q^{-n} ; c^{\prime} x q^{n-1}\right]_{1} \phi_{0}\left[q^{-m} ; y\right],
\end{aligned}
$$

summing the two ${ }_{1} \phi_{0}$, we get (4.11), which is $q$-analogue of a terminating version of a formula [3; Ex. 20(i) p. 102]. Lastly, we 
give $q$-analogues of reduction formulae for Appell's double, hypergeometric function:

$$
\begin{aligned}
& \phi\left[\begin{array}{c|c}
a: b, y ; c & x,-y ; q \\
c y: a ; a & -; 1 ;-
\end{array}\right]=\Pi\left[\begin{array}{c}
b x, y \\
x, c y
\end{array}\right]_{2} \phi_{2}\left[\begin{array}{c}
b, c ; x y \\
a, b x
\end{array}\right], \\
& \phi\left(\begin{array}{l|l}
-: a, b ; \frac{c}{a}, \frac{c}{b} & x, \frac{a b y}{c} ; q \\
c: \frac{a b y}{c} ;- & 1 ;-; 1
\end{array}\right)=\frac{[y]_{\infty}}{\left[\frac{a b y}{c}\right]_{\infty}{ }_{\infty}} \phi_{2}\left[\begin{array}{l}
a, b,-\frac{x}{y} ; y \\
c, 0
\end{array}\right], \\
& \phi\left[\begin{array}{l|l}
-: a, \frac{q}{a} ; c, \frac{q}{c} & -x, x ; q \\
d:-q ;-q & -;-;-
\end{array}\right]={ }_{6} \phi_{5}\left[\begin{array}{l}
a c, \frac{q^{2}}{a c}, \frac{a q}{c}, \frac{c q}{a}, 0,0 ; q^{2} ; x^{2} \\
q,-q,-q^{2}, d, d q
\end{array}\right] \\
& -\frac{x(a-c)(q-c a)}{a c(1-d)\left(1-q^{2}\right)} \cdot{ }_{6} \phi_{5}\left[\begin{array}{l}
a c q, \frac{q^{3}}{a c}, \frac{a q^{2}}{c}, \frac{c q^{2}}{a}, 0,0 ; q^{2} ; x^{2} \\
-q^{2}, q^{3},-q^{3}, d q, d q^{2}
\end{array}\right], \\
& \phi\left[\begin{array}{l|l}
a: b,-b ; c,-c \mid x,-x ; q \\
-: b^{2} ; c^{2} & -;-;-
\end{array}\right] \\
& ={ }_{6} \phi_{5}\left[\begin{array}{l}
a, a q, b c,-b c, b c q,-b c q ; q^{2} ; x^{2} \\
b^{2} q, c^{2} q, b^{2} c^{2}, 0,0
\end{array}\right] .
\end{aligned}
$$

Proof of (4.15). Rewrite the left hand side of (4.15) in the form

$$
\begin{aligned}
S & =\sum_{m=0}^{\infty} \frac{[b]_{m}[y]_{m} x^{m}}{[q]_{m}[c y]_{m}}{ }_{2} \phi_{2}\left[\begin{array}{l}
a q^{m}, c ; y \\
a, c y q^{m}
\end{array}\right] \\
& =\frac{[y]_{\infty}}{[c y]_{\infty}} \sum_{m=0}^{\infty} \frac{[b]_{m} x^{m}}{[q]_{m}}{ }_{2} \phi_{1}\left[\begin{array}{c}
q^{-m}, c ; y q^{m} \\
a
\end{array}\right] \\
& =\frac{[y]_{\infty}}{[c y]_{\infty}} \sum_{n=0}^{\infty} \frac{[b]_{n}[c]_{n}(-x y)^{n} q^{n / 2(n-1)}}{[q]_{n}[a]_{n}}{ }_{1} \phi_{0}\left[b q^{n} ;-; x\right] .
\end{aligned}
$$

Summing the inner ${ }_{1} \phi_{0}$ of (4.19) and we get (4.15) which is a $q$ analoque of $[3 ; \S 9.5-(7)]$ to which it reduces if we replace $a, b, c$ by $q^{a}, q^{b}, q^{c}$ respectively and let $q \rightarrow 1$.

Proof of (4.16). Left hand side of (4.16) may be rewritten as:

$$
\begin{aligned}
& S=\sum_{m=0}^{\infty} \frac{[a]_{m}[b]_{m} x^{m} q^{m / 2(m-1)}}{[q]_{m}[c]_{m}\left[\frac{a b y}{c}\right]_{m}}{ }_{2} \phi_{1}\left[\begin{array}{l}
\frac{c}{a}, \frac{c}{b} ; \frac{a b y}{c} q^{m} \\
c q^{m}
\end{array}\right] \\
& =\frac{[y]_{\infty}}{\left[\frac{a b y}{c}\right]_{\infty}} \sum_{m=0}^{\infty} \frac{[a]_{m}[b]_{m} x^{m} q^{m / 2(m-1)}}{[q]_{m}[c]_{m}}{ }_{2} \phi_{1}\left[\begin{array}{c}
a q^{m}, b q^{m} ; y \\
c q^{m}
\end{array}\right]
\end{aligned}
$$




$$
=\frac{[y]_{\infty}}{\left[\frac{a b y}{c}\right]_{\infty}} \sum_{n=0}^{\infty} \frac{[a]_{n}[b]_{n} y^{n}}{[q]_{n}[c]_{n}}{ }_{1} \phi_{0}\left[q^{-n} ;-;-\frac{x}{y} q^{n}\right] .
$$

Summing the inner ${ }_{1} \phi_{0}$ of (4.20) and we get (4.16). It is a $q$-analogue of $[3 ; \S(9.5)-(5)]$.

Proof of (4.17). The summation theorem [10]

$$
{ }_{4} \phi_{3}\left[\begin{array}{l}
a, \frac{q}{a},-q^{-n}, q^{-n} ; q \\
-q, c, \frac{1}{c} q^{1-2 n}
\end{array}\right]=\frac{\left[a c ; q^{2}\right]_{n}\left[\frac{c q}{a} ; q^{2}\right]_{n}}{[c]_{2 n}},
$$

could be written in the symmetrical form (on replacing $c$ by $c q^{-n}$ ):

$$
\begin{gathered}
\sum_{r=0}^{n} \frac{[a]_{r}\left[\frac{q}{a}\right]_{r}[c]_{n-r}\left[\frac{q}{c}\right]_{n-r}(-)^{r}}{\left[q^{2} ; q^{2}\right]_{r}\left[q^{2} ; q^{2}\right]_{n-r}} \\
=\frac{\left[a c q^{-n} ; q^{2}\right]_{n}\left[\frac{c}{a} q^{1-n} ; q^{2}\right]_{n}(-)^{n} q^{n / 2(n+1)}}{c^{n}\left[q^{2} ; q^{2}\right]_{n}},
\end{gathered}
$$

multiplying on both sides of (4.22) by $x^{n} /[d]_{n}$ and summing with respect to $n$ form 0 to $\infty$, we get (4.17) (on separating the even and odd parts on right hand side) on some simplification.

Proof of (4.18). The $q$-analogue of Watson's theorem [2] can be written in the symmetrical form (on replacing $b$ by $q^{1 / 2-n} / b$ ):

$$
\sum_{r=0}^{2 n} \frac{\left[c^{2} ; q^{2}\right]_{r}\left[b^{2} ; q^{2}\right]_{2 n-r}(-)^{r}}{[q]_{r}[q]_{2 n-r}\left[c^{2}\right]_{r}\left[b^{2}\right]_{2 n-r}}=\frac{\left[b^{2} c^{2} ; q^{2}\right]_{2 n}}{\left[b^{2} q ; q^{2}\right]_{n}\left[c^{2} q ; q^{2}\right]_{n}\left[b^{2} c^{2} ; q^{2}\right]_{n}\left[q^{2} ; q^{2}\right]_{n}},
$$

multiplying both sides of (4.23) by $[a]_{2 n} x^{2 n}$ and summing with respect to $n$ from 0 to $\infty$, we get

$$
\begin{aligned}
& \sum_{n=0}^{\infty}[a]_{2 n} x^{2 n} \sum_{r=0}^{2 n} \frac{\left[c^{2} ; q^{2}\right]_{r}\left[b^{2} ; q^{2}\right]_{2 n-r}(-)^{r}}{[q]_{r}[q]_{2 n-r}\left[c^{2}\right]_{r}\left[b^{2}\right]_{2 n-r}} \\
&={ }_{6} \phi_{5}\left[\begin{array}{c}
a, a q, b c,-b c, b c q,-b c q ; q^{2} ; x^{2} \\
b^{2} q, c^{2} q, b^{2} c^{2}, 0,0
\end{array}\right] .
\end{aligned}
$$

But, since

$$
\sum_{r=0}^{2 n+1} \frac{\left[c^{2} ; q^{2}\right]_{r}\left[b^{2} ; q^{2}\right]_{2 n+1-r}(-)^{r}}{[q]_{r}[q]_{2 n+1-r}\left[c^{2}\right]_{r}\left[b^{2}\right]_{2 n+1-r}}=0,
$$

therefore (4.24) yields 


$$
\begin{aligned}
\sum_{n=0}^{n}[a]_{n} x^{n} \sum_{r=0}^{\infty} \frac{\left[c^{2} ; q^{2}\right]_{r}\left[b^{2} ; q^{2}\right]_{n-r}(-)^{r}}{[q]_{r}[q]_{n-r}\left[c^{2}\right]_{r}\left[b^{2}\right]_{n-r}} \\
={ }_{6} \dot{\phi}_{5}\left[\begin{array}{c}
a, a q, b c,-b c, b c q,-b c q ; q^{2} ; x^{2} \\
b^{2} q, c^{2} q, b^{2} c^{2}, 0,0
\end{array}\right] .
\end{aligned}
$$

Now, rearranging the series in left hand side of (4.25), we get (4.18), (4.17) and (4.18) are the $q$-analogues of (4.2) and (4.4) of [4] respectively.

I am grateful to Dr. Arun Verma for suggesting the problem and for his helpful discussions during the preparation of this paper.

\section{REFERENCES}

1. G. E. Andrews, Summations and transformations for basic Appell series, J. London Math. Soc., (2), 4 (1972), 618-622.

2. On q-analogues of the Watson and Whipple summations, SIAM J. Math. Anal., 7 (1976), 332-336.

3. W. N. Bailey, Generalized Hypergeometric Series, Cambridge, 1935.

4. - On the sum of a terminating ${ }_{3} F_{2}(1)$, Quart. J. Math. (Oxford) (2), 4 (1953), $237-240$.

5. J. L. Burchnall and T. W. Chaundy, Expansions of Appells' double hypergeometric functions, Quart. J. Math. (Oxford), 11 (1940), 249-270.

6. Expansions of Appells' double hypergeometric functions II, Quart. J. Math. (Oxford), 12 (1941), 112-128.

7. F. H. Jackson, Transtormations of q-series, Mess. Math., 39 (1910), 145-153.

8. On basic double hypergeometric functions, Quart J. Math. (Oxford), 13 (1942), 69-82.

9. - Basic double hypergeometric functions II, Quart. J. Math. (Oxford), 15 (1944), 49-61.

10. V. K. Jain, Some transformations of basic hypergeometric functions, (unpublished Manuscript).

11. D. B. Sears, On the transformation theory of basic hypergeometric functions, Proc. London Math. Soc., (2), 53 (1951), 158-180.

12. L. J. Slater, Generalized Hypergeometric Functions, Cambridge University Press, 1966 .

13. M. Upadhyay, Certain transformations for basic double hypergeometric functions of higher order, Indian J. Pure and Applied Math., 4 (1973), 341-354.

14. A. Verma, Certain summation formulae for basic hypergeometric series, Canad. Math. Bull., 20 (3) (1977), 369-375.

Received November 13, 1978 and in revised form January 8, 1980.

UNIVERSITY OF ROORKEE

ROORKEE (U.P.) INDIA 



\section{PACIFIC JOURNAL OF MATHEMATICS}

\section{EDITORS}

DONALD BABBITT (Managing Editor)

University of California

Los Angeles, CA 90024

Hugo RossI

University of Utah

Salt Lake City, UT 84112

C. C. MOORE and ANDREW OGG

University of California

Berkeley, CA 94720

\section{J. DugundjI}

Department of Mathematics

University of Southern California

Los Angeles, CA 90007

R. FinN and J. Milgram

Stanford University

Stanford, CA 94305

\section{ASSOCIATE EDITORS}
E. F. BeCKENBACH
B. H. Neumann
F. WoLF
K. YoSHIDA

\section{SUPPORTING INSTITUTIONS}

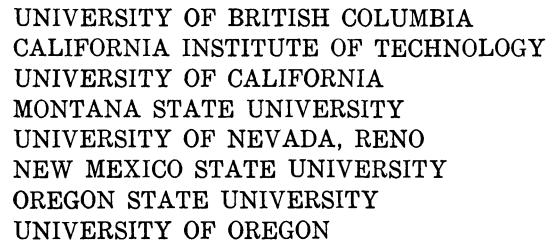

UNIVERSITY OF BRITISH COLUMBIA CALIFORNIA INSTITUTE OF TECHNOLOGY UNIVERSITY OF CALIFORNIA MONTANA STATE UNIVERSITY UNIVERSITY OF NEVADA, RENO NEW MEXICO STATE UNIVERSITY OREGON STATE UNIVERSITY UNIVERSITY OF OREGON

\author{
UNIVERSITY OF SOUTHERN CALIFORNIA \\ STANFORD UNIVERSITY \\ UNIVERSITY OF HAWAII \\ UNIVERSITY OF TOKYO \\ UNIVERSITY OF UTAH \\ WASHINGTON STATE UNIVERSITY \\ UNIVERSITY OF WASHINGTON
}

The Supporting Institutions listed above contribute to the cost of publication of this Journal, but they are not owners or publishers and have no responsibility for its content or policies.

Mathematical papers intended for publication in the Pacific Journal of Mathematics should be in typed form or offset-reproduced, (not dittoed), double spaced with large margins. Please do not use built up fractions in the text of the manuscript. However, you may use them in the displayed equations. Underline Greek letters in red, German in green, and script in blue. The first paragraph or two must be capable of being used separately as a synopsis of the entire paper. Please propose a heading for the odd numbered pages of less than 35 characters. Manuscripts, in triplicate, may be sent to any one of the editors. Please classify according to the scheme of Math. Reviews, Index to Vol. 39. Supply name and address of author to whom proofs should be sent. All other communications should be addressed to the managing editor, or Elaine Barth, University of California, Los Angeles, California, 90024.

50 reprints to each author are provided free for each article, only if page charges have been substantially paid. Additional copies may be obtained at cost in multiples of 50 .

The Pacific Journal of Mathematics is issued monthly as of January 1966. Regular subscription rate: $\$ 84.00$ a year (6 Vols., 12 issues). Special rate: $\$ 42.00$ a year to individual members of supporting institutions.

Subscriptions, orders for numbers issued in the last three calendar years, and changes of address shoud be sent to Pacific Journal of Mathematics, P.O. Box 969, Carmel Valley, CA 93924, U.S.A Old back numbers obtainable from Kraus Periodicals Co., Route 100, Millwood, NY 10546.

PUBLISHED BY PACIFIC JOURNAL OF MATHEMATICS, A NON-PROFIT CORPORATION

Printed at Kokusai Bunken Insatsusha (International Academic Printing Co., Ltd.). 8-8, 3-chome, Takadanobaba, Shinjuku-ku, Tokyo 160, Japan. 


\section{Pacific Journal of Mathematics}

\section{Vol. 91, No. 2 December, 1980}

Victor P. Camillo and Julius Martin Zelmanowitz, Dimension modules ... . . 249

Yonina S. Cooper, Stable sequences in pre-abelian categories ........... 263

Chandrakant Mahadeorao Deo and H. Ship-Fah Wong, On Berry-Esseen approximation and a functional LIL for a class of dependent random fields.........................................

H. P. Dikshit and S. N. Dubey, $|C, 1|$ summability of series associated with

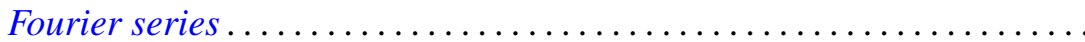

M. Edelstein, On the homomorphic and isomorphic embeddings of a semiflow into a radial flow.

Gilles Godefroy, Compacts de Rosenthal ..................... 293

James Guyker, Commuting hyponormal operators ................ 307

Thomas Eric Hall and Peter R. Jones, On the lattice of varieties of bands of

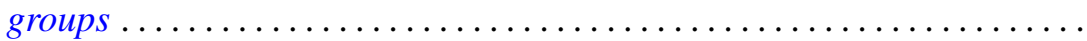

Taqdir Husain and Saleem H. Watson, Topological algebras with orthogonal Schauder bases ....................................

V. K. Jain, Some expansions involving basic hypergeometric functions of two variables. . .

Joe W. Jenkins, On group actions with nonzero fixed points ........... 363

Michael Ellsworth Mays, Groups of square-free order are scarce ........ 373

Michael John McAsey, Canonical models for invariant subspaces... 377

Peter A. McCoy, Singularities of solutions to linear second order elliptic partial differential equations with analytic coefficients by approximation methods...

Terrence Millar, Homogeneous models and decidability.

Stephen Carl Milne, A multiple series transformation of the very well poised

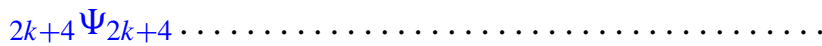

Robert Olin and James E. Thomson, Irreducible operators whose spectra are spectral sets...

Robert John Piacenza, Cohomology of diagrams and equivariant singular

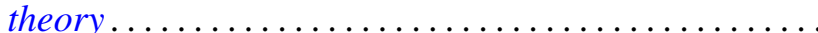

Louis Jackson Ratliff, Jr., Integrally closed ideals and asymptotic prime divisors

Robert Breckenridge Warfield, Jr., Cancellation of modules and groups and stable range of endomorphism rings.................

B. J. Day, Correction to: "Locale geometry" ...............

Stanley Stephen Page, Correction to: "Regular FPF rings" ... 